\title{
How the Autonomic Nervous System and Driving Style Change With Incremental Stressing Conditions During Simulated Driving
}

\author{
Antonio Lanatà, Member, IEEE, Gaetano Valenza, Member, IEEE, Alberto Greco, Claudio Gentili, \\ Riccardo Bartolozzi, Francesco Bucchi, Francesco Frendo, and Enzo Pasquale Scilingo, Member, IEEE
}

\begin{abstract}
This paper reports on the autonomic nervous system (ANS) changes and driving style modifications as a response to incremental stressing level stimulation during simulated driving. Fifteen subjects performed a driving simulation experiment consisting of three driving sessions. Starting from a first session where participants performed a steady motorway driving, the experimental protocol includes two additional driving sessions with incremental stress load. More specifically, the first stressing load consists of randomly administering mechanical stimuli to the vehicle during a steady motorway driving by means of a series of sudden and unexpected skids, such as those produced by a strong wind gust. These skids were supposed to produce in the driver a given level of stress. In order to assess this mental workload, dedicated psychological tests were performed. The second stressing load implied an incremental psychological load, consisting of a battery of time pressing arithmetical questions, added to the mechanical stimuli. For the whole experimental session, the driver's physiological signals and the vehicle's mechanical parameters were recorded and analyzed. In this paper, the ANS changes were investigated in terms of heart rate variability, respiration activity, and electrodermal response along with mechanical information such as that coming from steering wheel angle corrections, velocity changes, and time responses. Results are satisfactory and promising. In particular, significant statistical differences were found among the three driving sessions with an increasing stress level both in ANS responses and mechanical parameter changes. In addition, a good recognition of these sessions was carried out by pattern classification algorithms achieving an accuracy greater than $90 \%$.
\end{abstract}

Index Terms-Electrodermal response (EDR), heart rate variability (HRV), respiration activity (RSP), simulated driving, stress recognition, vehicle parameters.

Manuscript received May 23, 2013; revised March 21, 2014, June 5, 2014, and August 1, 2014; accepted October 17, 2014. The Associate Editor for this paper was C. Wu.

A. Lanatà, G. Valenza, A. Greco, and E. P. Scilingo are with the "E. Piaggio" Research Center and Department of Information Engineering, University of Pisa, 56122 Pisa, Italy (e-mail: a.lanata@centropiaggio.unipi.it).

C. Gentili is with the Department of Surgical, Medical, Molecular and Critical Area, Clinical Psychology Chair, University of Pisa, 56124 Pisa, Italy.

R. Bartolozzi is with the Institute for Structural Durability and System Reliability, Fraunhofer LBF, 64289 Darmstadt, Germany.

F. Bucchi and F. Frendo are with the Department of Mechanical, Nuclear and Production Engineering, University of Pisa, 56122 Pisa, Italy.

Color versions of one or more of the figures in this paper are available online at http://ieeexplore.ieee.org.

Digital Object Identifier 10.1109/TITS.2014.2365681

\section{INTRODUCTION}

$\mathbf{T}$ HE EFFECT of the so-called stress has received great interest from the scientific community during the last decades. Even if a first definition of stress was done in 1936 by Selye [1], the research literature currently reflects wide and divergent opinions concerning stress [2]. For the sake of clarity, we adopted the first definition proposed by Selye, where stress is considered an adaptation phenomenon as a response to an external event. Here, we are interested in studying how the driver response, in terms of autonomic nervous system (ANS), changes and how driving style is modified by an incremental stressing protocol. The literature has well noted how stressing stimulation produces human response, which incorporates physiological, cognitive, behavioral, and emotional dimensions [3]. Starting from the findings in [4]-[7], which assert that each individual has response components within a greater somatovisceral response organization that directs resource allocation based on situational circumstances, in this work we investigate how the administered stressing stimuli can produce not only ANS changes but also a modification of the driver behavior and a variation of the driving style. Indeed, ANS activity has been largely characterized by means of peripheral signals such as heart rate variability (HRV) [8]-[13], respiration activity (RSP) [14]-[16], and electrodermal response (EDR) [17], [18], and since stress has been also one of the leading causes of cardiorespiratory, gastrointestinal, dermatological, and neurological diseases, we studied whether the same pattern of signals can be considered as an indirect biomarker of stress. The mentioned signals, in fact, can be easily and noninvasively monitored in actual environments with wearable systems [19], [20] such as sensorized t-shirts [5], [21], [22] and fabric gloves [23], [24]. Here, an incremental stressing protocol was administered by means of two stressors. More precisely, at the beginning, a series of sudden and unexpected skids was applied to the vehicle and, afterward, an additional psychological stressor, i.e., a series of time-pressing arithmetical questions, was introduced. In this paper, we propose an approach to automatically recognize the two different stress-level phases, starting from a baseline acquisition. The driving sessions were performed by a complete driving simulator developed at University of Pisa (UNIPI) [25], in which a 14-degree-of-freedom vehicle model was implemented. A simulator host control station permitted to alter in real time the vehicle dynamics and the driving scenario by randomly inducing lateral skids during simulated drives. 
Moreover, subject driving style was inferred from longitudinal speed and steering wheel angle of the vehicle. According to the current state of the art, we studied the effect of the stressors in terms of physiological and mechanical multidimensional feature space. A statistical analysis was performed to detect the most significant features, and afterward, an automatic classification algorithm was trained in order to test whether the feature patterns related to different stress levels are distinguished. The underlying hypothesis was that by increasing the stress level, the ANS patterns and the driving style change accordingly. Moreover, in order to better investigate the sympathovagal balance in the two different stress-level conditions, a detailed analysis of the EDR parameters was carried out. EDR, indeed, is strongly related to sympathetic activity [26], and reasonably, it could be profitably exploited, in a future perspective, in actual driving monitoring.

Section II reports evidences from the literature about how stress can be interpreted and evaluated in several conditions. In addition, the ambiguity of the stress definition over the years was described as well, along with the relationship between stress and mental workload, stress and attention, and stress and ANS derived measures. In Section IV, the experimental protocol along with the psychological evaluation of the participants is reported. In Section IV-B, the simulator prototype from the University of Pisa used in this study is briefly presented and described. Finally, in Section IV-C, the processing chain to analyze physiological and vehicle parameters is described. The processing techniques used to extract features from each mechanical and physiological signal are reported in Section IV-D. The statistical analysis on feature distributions and the pattern recognition strategy used to analyze the stressful driving sessions can be found in Section IV-E. Section V presents the obtained experimental results, and finally, Section VI reports on the discussion and conclusion of this work, noting the impact that such a study could have in stress management, particularly in the specific automotive field.

\section{BACKGROUND}

The literature dealing with stress has noted not only the strong ambiguity of the stress definition but also that there are no psychological stressors in absolute and objective sense [2]. In this paper, we refer to the first stress definition given by Selye [1]; therefore, the label stressor can be applied to any task that requires mental and/or physical resources. It is widely accepted that task demand triggers a psychological response such as frustration, anxiety, or psychological discomfort [22], [27]. This response often contains both physiological and mental components that compete for resources, acting as a secondary workload factor drawing resources away from the primary demand, devoting them instead to secondary psychological processes. Stress involves several human aspects (e.g., behavioral or emotional), and it is strongly dependent on the amount of resources that can be recruited in a task demand. The resource recruitment process that produces a behavioral response is due to an arousal involvement in the activation process [28]. Several works in the literature define the attention process as a reduction in resources focused on peripheral tasks, centralizing their focus on main tasks [29]. Main and peripheral tasks appear to depend on whichever stimulus the subject perceived as the most salient [30]. This process can either enhance or reduce performance, depending on the task and the situation [31]. Stress or workload can modify this process. Backs [32] modeled physiological markers of workload (i.e., heart rate (HR) and respiration period). Cacioppo and Tassinary addressed the potential problems that exist in linking physiological and other data together in causal relationships [33]. They caution that when there is a relationship between a process or event and a concomitant physiological change, there are numerous possible causal explanations. Hancock et al. explored various measurement methodologies of mental workload, supposing that mental workload affects the activity of the central nervous system [34]. Many researchers explored attentional workload associated with driving a car. They found that under nonstress conditions, driver attention was focused on objects unrelated to the driving task $30 \%-50 \%$ of the time [35]. This finding may provide some descriptive evidence of how drivers discard tasks when cognitively overloaded; these results are not universal across the research literature but do reflect most of the findings [36]. Fernandez and Picard [37] characterized the stress during driving by asking math questions with different velocities. Healey and Picard [38] discriminated several stress levels by processing the mentioned ANS signals during real driving, relying on the fact that different stress levels could be linked to different kinds of streets. Other studies were performed by testing the effect of alcohol [39], sleep deprivation [40], or cellphone conversation [41]. Matthews et al. in [42] also studied drivers under various conditions of stress (increased workload via a grammatical reasoning task using both visual and auditory inputs). In this instance, drivers adapted to higher levels of demand efficiently. Generally, mental task load resulted in internal distraction affecting the attentional process (e.g., for visual stimuli, a reduction in the number of targets scanned has been observed). Studies based on performance changes as a consequence of lack of attention in car drivers, due to either abnormal psychophysical conditions or distraction, have been carried out often using the perception response time (PRT). PRT is usually achieved by specific tests accomplished while driving [43]. Specifically, drivers' fatigue and hypovigilance due to sleep deprivation and/or alcohol assumption were investigated by a braking response test [44], and driving capabilities in patients with sleep apnea were assessed by a reaction test [45]. In addition, lateral skid tests have been employed in different ways, focusing, in general, on the response to the steering wheel. Methods and results of such tests are summarized in [46] and [47], whereas a detailed analysis of several test parameters of a lateral skid test due to a side gust has been investigated in [48]. A methodology to investigate a driver's response and ability was proposed in [25]. The driver's attention has been widely investigated also by analyzing the driver performance in standard driving conditions (i.e., without specific perception-response tests). In these cases, the deviation of the driver's behavior from a target is typically assessed by the deviation of the longitudinal speed, steering wheel angle, and lateral position of the car in the lane [44], [49]. Finally, it is worth noting that physiological signal analysis is not completely new in the stress evaluation, 
and some evidences have been already shown a good stress characterization with the frequency-domain parameters of the HRV [50]. Specifically, a significant increase in the LF/HF ratio has been reported in healthy subjects undertaking stressful tasks [51] and in patients with posttraumatic stress disorder [52]. The major reason of such an increase seems to be a reduction in the parasympathetic activity [53]. Moreover, the EDR has been integrated with a distributed wireless sensor network for stress monitoring and detection [54], [55], and the major results have been obtained when the EDR information was integrated with the HRV one [56], [57]. As a matter of fact, in the stress assessment for driving alertness, the HRV and the EDR have been already used as physiological measures for such an assessment [38], [58]. Moreover, latest research has been focused on how an altered emotional state can affect driving style, producing car accidents, and in this context, several groups developed systems to monitor and control the driver affective state. For example, Conjeti et al. [59] developed a safety-critical wearable computer capable of monitoring the affective state in drivers. In summary, they presented a perspective on the system design requirements and tradeoffs, architectural aspects, affective state diagnosis, and associated physiological signal processing for wearable driver assistance systems. In addition, Katsis et al. [60] developed a real-time wearable system for remote monitoring emotional state of car-racing drivers. This system, which is named the augmentation system for robust emotional understanding, consisted of a wearable device and a centralized unit. The system acquires selected biosignals, preprocesses them, and transmits them wirelessly from the subject site to the centralized system. Then, a tree-augmented naive Bayesian classifier was used to classify euphoria and dysphoria.

\section{Beyond the StATE OF THE ART}

In this paper, we propose a novel holistic approach to investigate about the driver behavior, implementing a multivariate and multimodal methodology. In particular, we acquired both the vehicle parameters and physiological response as well as the psychological evaluation of each subject. Moreover, this work aimed at verifying the hypothesis that the driving style is altered as a consequence of a stressed-driver status. As a matter of fact, here, we considered both the direct response to the stimuli and signal acquired in a successive time window, highlighting that the driving style and the physiological status remain altered in the whole session and, in addition, that only a differential analysis can appreciate this dynamics. Finally, we showed that an intelligence engine can be trained to detect an altered driver status with a high level of accuracy, sensitivity, and specificity.

\section{Materials And Methods}

Here, we describe the experimental protocol along with the driving simulator and the physiological acquisition system. Moreover, the methodology of signal processing and pattern recognition is reported.

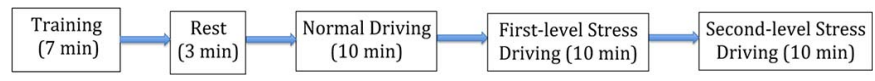

Fig. 1. General block scheme of the experimental protocol timeline.

\section{A. Experimental Protocol}

Fifteen healthy volunteers, aged $30 \pm 9$, were asked to perform simulated driving with the UNIPI prototype [25]. All of the enrolled participants underwent a psychological evaluation for both trait and state. More specifically, psychological state evaluation aimed at verifying whether the task induced a change in psychological state and an increase of stress and anxiety. Before starting the experimental session, the ZKPQ test was administered to the volunteers. It represents a five-factor (Impulsive Sensation Seeking, Neuroticism-Anxiety, AggressionHostility, Sociability, and Activity) personality inventory. The scope was to discard eventual subjects exhibiting disruptive personality disorders. More specifically, we were particularly interested in controlling the first two dimensions. The first is a factor related to impulsivity and inhibition of actions and might be a relevant confounding factor for driving skills. The latter is a dimension involved in anxiety and emotional regulation and dysregulation and might as well play a confounding role in stressful situations. The aim was to exclude those subjects who were significantly over the normative values of these two dimensions. However, no subject exceeded the normative thresholds; therefore, all were included in the study. The evaluation was performed asking participants to fill out the StateTrait Anxiety Inventory, version Y1 (STAI-Y1) [61]. This is a 20-item self-report scale measuring on a four-point Likert scale the degree of state anxiety. Since it measures anxiety in the present moment, it is often used to evaluate changes and anxiety perception after emotional and stressful engaging tasks. Currently, STAI-Y1 is widely used to assess changes in these subjective emotions and to verify at a subjective level the efficacy of the task in induce stress (e.g., [62]). Participants were asked to fill out the questionnaire twice: right before and right after the driving task. Our hypothesis was that the task in which the volunteers were involved increased their stress level. This assumption was assessed through changes in STAI-Y1 scores occurred between pre- and post-task. In addition, trait evaluation was performed as well. A trait can be thought of as a relatively stable characteristic that causes individuals to behave in certain ways. This evaluation was conducted to rule out the possible confounding effects of psychological traits on the stress response evoked by the task. In particular, in order to verify the general tendency to display an anxious emotion, the participants were asked to fill out the State-Trait Anxiety Inventory, version Y2 (STAI-Y2). The choice of simulated driving is justified by the possibility of having more effective and reliable trials decreasing the variability induced by real environments as suggested in [63], by means of a driving simulator that has been already proved to accurately replicate real driving scenarios [64]. Moreover, it is worth noting that none of the subjects had ever experienced simulated driving before, and nobody reported any psychopathological events in life. The experimental protocol was structured into the following five phases: training driving, rest, normal driving, first-level stress driving, and second-level 


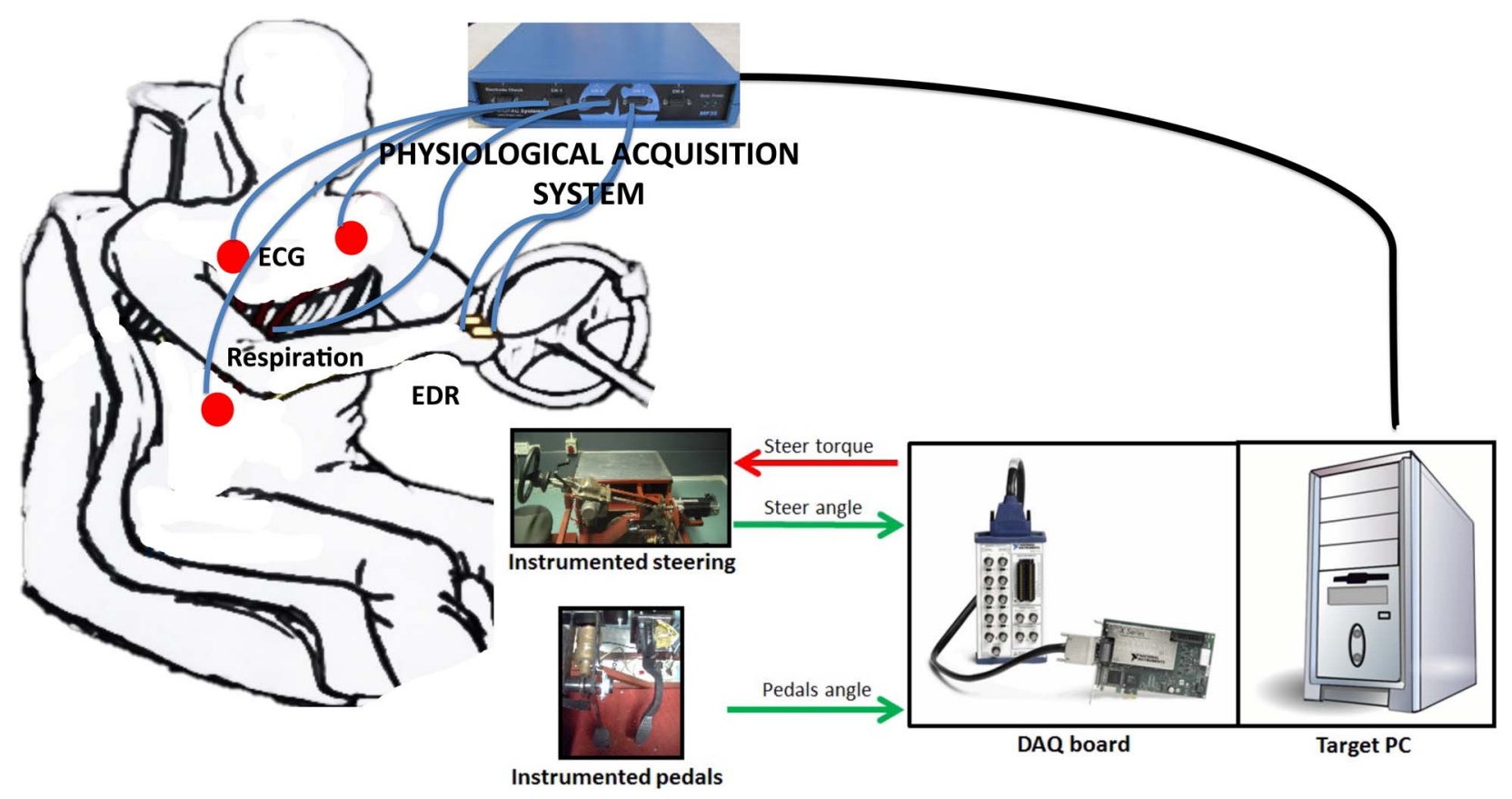

Fig. 2. Real-time vehicle model scheme and physiological system acquisition. Left top corner, physiological signal acquisition system and electrode placement for ECG, EDR, and respiration signal. Right bottom corner, both steering control and sensitized pedals are shown. On the right image side, a scheme of the real-time National Instrument acquisition system is presented.

stress driving (see Fig. 1). Along with all experimental sessions, several signals such as electrocardiogram (ECG), RSP, and EDR were continuously acquired. During the training phase, the subject experienced for the first time the simulated environment and was free to drive with no constraints. Then, the subject was asked to stop driving and keep the eyes closed at rest for 3 min while the car was still. This resting phase is aimed at detecting the physiological baseline of each subject. Then, three different driving sessions, where an incremental stress level was induced on each participant, started. During the first driving session (named normal-driving), the participant performed motorway driving, keeping a constant velocity of $100 \mathrm{~km} / \mathrm{h}$. As a matter of fact, this phase constitutes the first slow and continuous mechanical stimulation, without exogenous stressing events. The second driving session (named first-level stress session) was characterized by motorway driving during which a set of unpredictable lateral strong skids was artificially imposed to the car, by means of the host PC. Such a condition can occur in reality with a strong gust of wind, which requires an immediate reaction of the driver. Particularly, the controller superimposed a step in the lateral force with an amplitude of $10 \mathrm{kN}$ and a duration of $0.7 \mathrm{~s}$. The driver was asked to react trying to bring back the vehicle on its previous position on the lane as soon and as stable as possible. In the third driving session (named second-level stress session), a set of time-pressing mathematical questions (i.e., addition, subtraction, multiplication, and division) [37] was added to the lateral skids. The scope was to induce a mental load to the driver in order to verify whether it could provoke an alteration of performances. Indeed, mathematical questions are very widely used and validated tests to induce a stressful response [65]-[67]. Reaction times were estimated by means of a previously developed algorithm, whose details can be found in [25], for evaluating possible differences between first-level, second-level, and third-level stress sessions. Moreover, during all of the driving sessions, the driver was also asked to keep the car speed as constant as possible and as close as possible to a target value (set to $100 \mathrm{~km} / \mathrm{h}$ ), while applied lateral force was randomly induced, at least, once per minute, but with an interstimulus interval no less than $30 \mathrm{~s}$.

\section{B. Driving Simulator}

The system used during the driving tests is a fixed-base driving simulator that reproduces a medium-size European car with an automatic transmission. The driver interfaces are the steering wheel, the throttle pedal, and the brake pedal. They are installed into a real car cockpit chassis and are instrumented with rotational encoders (steering wheel) and linear potentiometers (throttle and brake pedal). A brushless motor is connected to the steering column and applies a resistant torque to the steering wheel, whereas the pedals are endowed with passive feedbacks. Moreover, encoders, potentiometers, and the brushless motor are interfaced to the target PC, wherein the vehicle model runs in real time (see Fig. 2). The driver perceives the vehicle motion conditions by means of the front view, the steering torque feedback, and the engine noise. The target PC communicates via a local area network (LAN) the position and the orientation of the car to the traffic generator PC wherein a scenario reproducing a motorway environment of about $35 \mathrm{~km}$ connected to an urban area is implemented. The driver's front view (see Fig. 3) is projected on a flat screen measuring about $2 \mathrm{~m}$ in width and $1.5 \mathrm{~m}$ in height. The driver's visual field of view results to be about $60^{\circ}$. On the screen, together with the road scenario, the car internal rearview mirror and a virtual dashboard are also represented. The dashboard gives to the driver information about the car speed and the engine speed. 


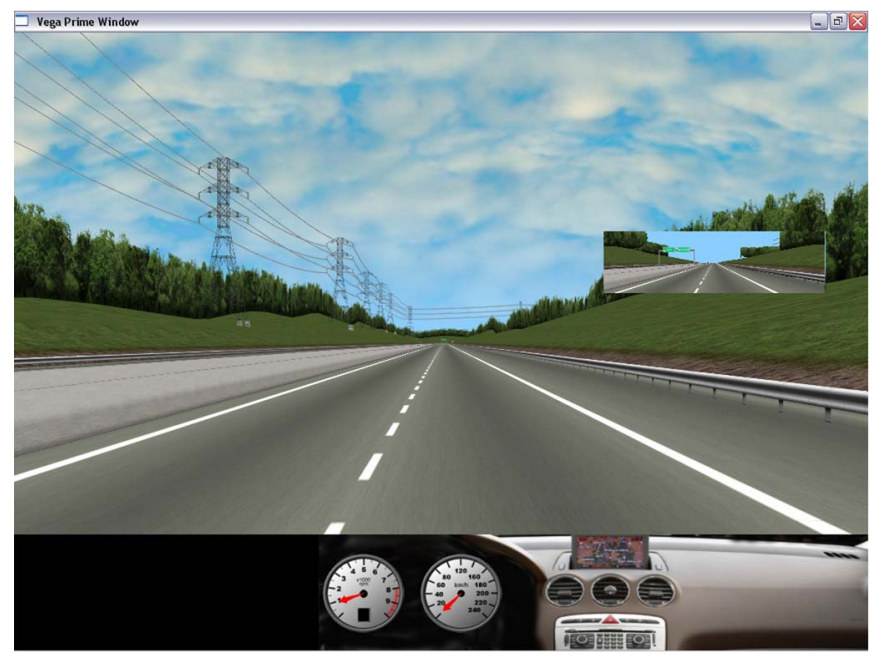

Fig. 3. Driver's front view.

The image of the front view is refreshed with a mean frequency of $30 \mathrm{~Hz}$, which slightly changes depending on the effort of the graphical software. Two additional computers are present in the driving simulator room, which interact via a LAN with the real-time simulation. The host PC manages and controls the simulation running in the target $\mathrm{PC}$ by means of a specific MATLAB toolbox (xPC Target). It receives and elaborates data, and it imposes modifications to the real-time running model (e.g., imposing steering, braking, etc.). The instructor station PC lets the operator control the vehicle behavior in the graphical scenario and succeeds in changing some environmental parameters, such as weather (rain, fog, etc.) and daylight. The vehicle model was developed in MATLAB/Simulink environment, and it is completely parametric, thus allowing for simulating several vehicle types. It is made of several analytic and differential blocks representing different vehicle subsystems (braking systems, engine, tires, steering system, etc.). The whole dynamic system is analytically solved by using a solver with a fixed step size of $2.5 \mathrm{~ms}$ (solver frequency: $400 \mathrm{~Hz}$ ). The fixed step, which is required for real-time applications, is about seven times greater than the task execution time, i.e., the time required by the target PC to solve a single time step, thus allowing for real-time simulation. The driving simulator acquires in real time and stores 60 signals concerning the vehicle motion condition and the main vehicle subsystems at $100-\mathrm{Hz}$ frequency.

\section{Physiological and Mechanical Signal Acquisition}

In this paper, three physiological signals (ECG, RSP, and EDR) were simultaneously acquired by using the BIOPAC MP150 with a sampling rate of $250 \mathrm{~Hz}$ for all of the signals. Each signal was segmented in nonoverlapped time windows whose length was in agreement with experimental protocol phases.

1) ECG: We used the ECG100C electrocardiogram amplifier from BIOPAC Inc., with a noise voltage of $0.1 \mu \mathrm{V}$ (RMS) in the bandwidth $0.05-35 \mathrm{~Hz}$, a CMRR of $110 \mathrm{~dB}$, a 16-bit ADC, and an amplitude resolution of $\pm 0.4 \mathrm{mV}$ to record the $\mathrm{D} 2$ lead ECG signal. Pregelled Ag/AgCl electrodes were placed following the Einthoven triangle configuration. Electrode placement is a crucial procedure to obtain reliable physiological measures [68], [69]. It has been demonstrated that when the electrode distance is more than $15 \mathrm{~cm}$ from the heart, they are considered equidistant [70]. Accordingly, we used three electrodes placed on the right arm, left arm, and left leg. One pair was selected to record, and the other one was used as a ground. Electrodes were all of silver-silver-chloride type with hydrogel spread uniformly. Cables were not twisted on themselves.

ECG signal was used to extract the HRV [10], which reflects the sympathetic-parasympathetic balance [71]. HRV refers to the variation of the time interval between consecutive heartbeats. The ECG was prefiltered through a moving-average filter (MAF) in order to extract and subtract the baseline. The frequency response of an $M$ point MAF is expressed as follows: $|H[f]|=\sin (M \pi f) / M \sin (\pi f)$. Accordingly, we have chosen $M=500$, which corresponds to a duration of $2 \mathrm{~s}$, in order to obtain a cutoff frequency of $0.5 \mathrm{~Hz}$, approximately. Since HRV refers to the change of in HR over time, a QRS complex detection algorithm was used. Automatic QRS detection can be addressed using several methods, depending on the characteristics of the specific ECG signal [72], e.g., signalto-noise ratio, signal power, and ECG leads. We adopted the automatic algorithm developed by Pan-Tompkins [73]. This algorithm allowed us to extract each QRS complex and to detect the corresponding R-peak. Hence, the RR interval $\left(t_{R-R}\right)$ is defined as the interval between two successive QRS complexes. Nevertheless, not all of the RR intervals obtained by the automatic QRS detection algorithm were correct. Any technical artifact (i.e., errors due to the R-peak detection algorithm) in the RR interval time series may interfere with the analysis of these signals. Therefore, an artifact correction was needed. In this paper, we adopted a proper piecewise cubic spline interpolation method [74], [75]. In addition to the technical ones, also physiological artifacts could be present in the analyzed RR series. They include ectopic beats and arrhythmic events. We manually checked for physiological artifacts, and only artifactfree sections have been included in the analysis.

2) RSP: An RSP100C respiration amplifier was dedicated to record the RSP, by using the piezoresistive sensor TSD201. Since the piezoresistive sensor changes its electrical resistance if stretched or shortened, it resulted to being directly proportional to thoracic circumference variations [14]. It was processed to detrend the baseline and reject the movement artifacts. Baseline was obtained and removed by means of the MAF technique as previously described for the ECG signal treatment. In addition, the RSP was limited in the bandwidth by using a tenth-order low-pass finite impulse response filter with a cutoff frequency of $1 \mathrm{~Hz}$ approximated by Butterworth polynomial.

3) EDR: EDR was acquired by a GSR100C amplifier through two $\mathrm{Ag} / \mathrm{AgCl}$ electrodes placed on the index and middle fingertips. EDR is counted as the ratio between an imposed continuos voltage of $0.5 \mathrm{~V}$ between the two fingers and the passing current, and it is referred to changes in the electrical conductance of the skin, which are due to psychologically induced sweat gland activity [76], [77] after a sympathetic stimulation. Since EDR is a sweat gland activity measure and is directly controlled by sympathetic nerve activity [17], [78], it 
is considered as an ideal way to monitor the ANS. EDR can be acquired at the palmar and finger surface, because they are suitable anatomical sites of sweat eccrine glands. In the literature, many studies have indicated that electrodermal changes are associated to the arousal of the stimuli [79], [80]. Hereinafter, we refer to the EDR measurement as skin conductance (SC). SC is characterized by a tonic [i.e., SC level (SCL)] and a phasic component [SC response (SCR)]. SCL is the slowly varying baseline level of SC, and it is related to a person's overall state, whereas SCR is consider event related, and it comes up within a predefined response window after a given stimulus (1-5 s) [81]. When the interstimulus time interval is shorter than SCR recovery time, consecutive SCRs can be overlapped. This issue is one of the main problems in the SC analysis, because it does not allow for a correct decomposition into the phasic and tonic components. In this paper, we adopted a deconvolution model, which allows directly estimating the sudomotor nerve activity (SMNA) being the controller of the eccrine sweat gland activity [82]. The SMNA shows an interstimulus time interval shorter than SCR signal avoiding the overlapping problem. Specifically, the SC is the result of a convolution model between SMNA and the following biexponential impulse response function (IRF), which is named Bateman function [83]:

$$
\operatorname{IRF}(t)=\left(e^{-\frac{t}{\tau_{1}}}-e^{-\frac{t}{\tau_{2}}}\right) \cdot u(t)
$$

where $u(t)$ is the stepwise function. The steepness of onset and recovery is determined by time constants $\tau_{1}$ and $\tau_{2}$. The Bateman function describes a bicompartment model of the diffusion process of the sweat through the sweat ducts (first compartment) and the stratum corneum (second compartment) [84].

\section{Mechanical and Physiological Features}

1) Mechanical Features: Two groups of mechanical parameters can be extracted from the vehicle during the simulated driving. The first refers to parameters calculated within a small transient right after each lateral skid, which lasted for $5 \mathrm{~s}$, whereas the second group is estimated over periods between two consecutive skids when the transient is over. Indeed, the mean and standard deviation of the time windows, during which the second group was computed, were of $43.36 \pm 16.44 \mathrm{~s}$. The first class of features can be considered as short-time response to a skid, whereas the second one are indexes of long-term steady driving. In Fig. 4, a typical plot of the steering signal during the lateral skid is presented, and the main detectable signal attributes are highlighted. In detail, the following parameters were considered:

- $t_{r}$ : reaction time, i.e., the time from the step of lateral force to the first peak of the steering wheel angle;

- $t_{h}$ : first response time, i.e., time from the first peak to the second (negative) peak of the steering wheel signal;

- $t_{a}$ : ability time, i.e., time from the first reaction to the end of maneuver, defined as the instant when the steering wheel angle entries stable in a range $\left( \pm 5^{\circ}\right)$;

- $\delta_{\max , 1}$ : first peak value of the steering wheel angle;

- $\delta_{\max , 2}$ : second peak value of the steering wheel angle;

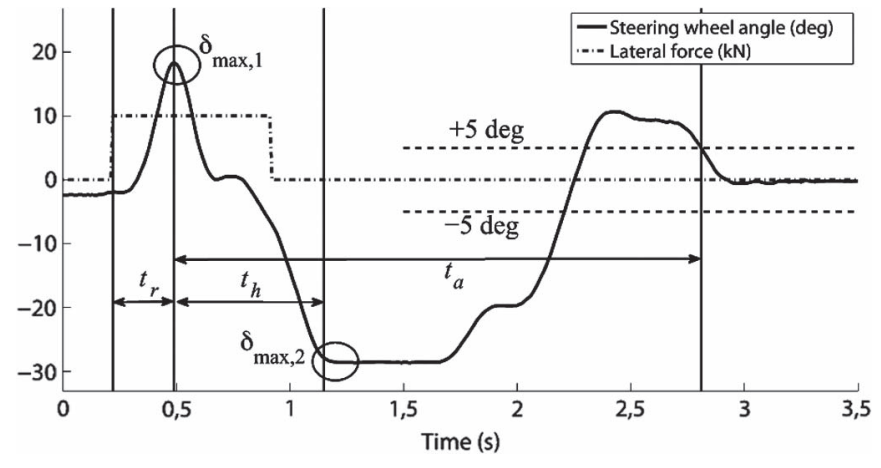

Fig. 4. Steering wheel angle and lateral force during the skid.

- $A U C$ : total area under the curve of the steering wheel signal from the beginning to the end of the maneuver;

- $N r_{\text {sign }}$ : number of sign changes of the steering wheel signal from the beginning to the end of the maneuver.

In steady driving conditions, the driver target consisted in maintaining constant velocity while driving on a motorway right lane. The steering wheel angle $\delta_{A}$ and the vehicle speed $V_{A}$ were recorded. Then, concerning the steering angle, the difference $\triangle_{S}$ between the acquired signal $\delta_{A}$ and the ideal target profile $\delta_{T}$ was computed, i.e.,

$$
\triangle_{S}=\left|\delta_{A}-\delta_{T}\right|
$$

The ideal target steering wheel angle was obtained for each driver as the averaged steering wheel angle, over a properly defined track length, of the order of $30 \mathrm{~m}$. In the same way, the difference $\triangle_{V}$ between the acquired speed $V_{A}$ and the target one $V_{T}$, which was kept constant at $100 \mathrm{~km} / \mathrm{h}$ along the whole path, is defined as

$$
\triangle_{V}(x, y)=\left|V_{A}-V_{T}\right| .
$$

The mean value and standard deviation of the steering wheel angle difference $\triangle_{S}$ and the speed difference $\triangle_{V}$ were computed over time periods between two consecutive skids. During the reaction test, a previously developed specific tool [25], consisting of a lateral skid response test that can occur with a sudden loss of grip or a strong gust of wind, was performed. All these parameters are typically calculated during the study of simulated driving, as reported in [25], [44], and [49].

2) Physiological Features: Physiological features have been extracted from ECG, RSP, and EDR. As the transient after the skid stimulus is too short to calculate features from ECG and RSP, they were extracted only in the time intervals between two consecutive skids. EDR, instead, has two components that, by definition, can be calculated in the 5-s transient intervals (phasic) as well as during steady phases (tonic). Feature extraction methods were applied in the time and frequency domains (statistics, geometrics). ECG, RSP, and tonic features were computed in time windows having mean value and standard deviation of $43.36 \pm 16.44 \mathrm{~s}$.

HRV [10] is related to the time intervals between heartbeats. Several features were extracted in both time and frequency domains. In the time domain, we extracted statistical parameters and morphological indexes. Fixing a time window (NN), the 
extracted features were simple MNN and SDNN, which are the mean value and the standard deviation of the $\mathrm{NN}$ intervals, respectively; the root mean square of successive differences of intervals and the number of successive differences of intervals, which differ by more than $50 \mathrm{~ms}$ (pNN50\% expressed as a percentage of the total number of heartbeats analyzed). Morphological or geometrical HRV analysis relies to a conversion of the series of NN intervals into a geometric pattern such as the sample density distribution of $\mathrm{NN}$ interval durations, sample density distribution of differences between adjacent NN intervals, and to analyze the variability by geometric and/or graphics properties of the resulting pattern. Here, we propose a triangular index. It is the integral of the density distribution (the number of all $\mathrm{NN}$ intervals) divided by the maximum of the density distribution. Triangular interpolation approximated the NN interval histogram by linear functions, the baseline width (expressed in seconds) of this approximation (base of the triangle) is used as HRV index. Referring to features extracted in the frequency domain, they were based on the power spectral density (PSD) of the HRV. In this paper, we estimated the PSD of HRV by using the autoregressive (AR) model, which provided a better frequency resolution than the nonparametric version. It is worth noting that transformations, in the frequency domain, based on the Fourier transform technique result in not being particularly suitable for analyzing nonstationary signals. Considering HRV as an output process $z(n)$ of a causal, allpole, discrete filter whose input is white noise, the AR method of order $p$ is expressed as

$$
z(n)=-\sum_{k=1}^{p} a(k) z(n-k)+w(n)
$$

where $a(k)$ are AR coefficients and $w(n)$ is white noise of variance equal to $\sigma^{2}$. The $\operatorname{AR}(p)$ model is characterized by $\mathrm{AR}$ parameters $\left\{a[1], a[2], \ldots, a[p], \sigma^{2}\right\}$. In this paper, we obtained high resolution in frequency and a stable AR model by using the Burg method, according to Akaike [85]. In the frequency domain, three main spectral components were distinguished in a spectrum calculated from short-term recordings: very low frequency (VLF), low frequency (LF), and high frequency (HF) components. It is well known, in the literature, that the distribution of the spectral power gives an indication of the ANS modulation. Current HRV research, in the frequency domain, analysis suggests that even though the frequency band division represents a unique and noninvasive tool for achieving an assessment of the autonomic function, the only use of HF and LF components are not able to precisely assess the state of sympathetic activation. Therefore, in addition to VLF, LF, and HF power, we also calculated the LF/HF ratio, which should give information about the sympathovagal balance [86].

$R S P$ signal is used to extract a group of features including respiration rate (RSPR), mean and standard deviation of the first (MFD and SDFD, respectively) and second derivative (MSD and SDSD, respectively), i.e., variation of the respiration signal, standard deviation of the breathing amplitude, and several other statistical parameters. RSPR was achieved as the maximum magnitude value of the frequency spectrum. Statistical parameters were calculated in order to characterize the differences
TABLE I

List of Mechanical and Physiological Features

\begin{tabular}{|c|c|}
\hline Feature set & Signals \\
\hline \hline MNN, SDNN, RMSSD, & HRV \\
pNN50, TINN & \\
VLF, LF, HF, LF/HF & \\
\hline Power in 0-0.1 Hz, 0.1-0.2 Hz, & RSP \\
0.2-0.3 Hz, 0.3-0.4 Hz, Central Frequency & \\
SEM, RSPR, MFD, SDFD, MSD, & \\
SDSD, SDBA, MAXRSP, MINRSP, & \\
DMMRSP, Skewness, Kurtosis & \\
\hline Lat, nSCR, Mean.SCR, Mean.Tonic, AmpSum, & EDR \\
AUC, Var.SCR, Var.Tonic & \\
\hline AUC, $N r_{\text {sign }}, \delta_{\max , 1}$, & Vehicle \\
$\delta_{\max , 2}, t_{r}, t_{h}, t_{a}$, & \\
$\triangle_{V}(x, y), \triangle_{S}$ & \\
\hline
\end{tabular}

between inspiratory and expiratory phases. These parameters included the maximum and the minimum value of breathing amplitude and their difference. In addition, we measured the asymmetries between the two respiratory phases by using highorder statistics. In detail, we calculated the third-order statistics (i.e., skewness), which takes into account the quantification of the asymmetry of the probability distribution, and the fourthorder statistics (i.e., kurtosis), which is a measure of the "peakedness" of the probability distribution. Moreover, another parameter was the standard error of the mean (SEM), which was calculated as $S E M=\sigma / \sqrt{n}$, where $\sigma$ is the standard deviation, and $n$ is the number of points within the window $W$. Concerning features in the frequency domain, the spectral power in the bandwidths $0-0.1 \mathrm{~Hz}, 0.1-0.2 \mathrm{~Hz}, 0.2-0.3 \mathrm{~Hz}$, and $0.3-0.4 \mathrm{~Hz}$ was also calculated [87].

$S C$ decomposition in its components was performed by means of Ledalab 3.2.2. software package for MATLAB. The signal was filtered by a low-pass zero-phase forward and reverse digital filter [88] with a cutoff frequency of $2 \mathrm{~Hz}$. In detail, SC data were described [82] as

$$
S C=\left(D R I V E R_{\text {tonic }}+D R I V E R_{\text {phasic }}\right) * I R F .
$$

The sum of the two driver functions, which represents the SMNA, was achieved by a deconvolution between the acquired $\mathrm{SC}$ and the Bateman function (IRF). The tonic and phasic driver components in (5) were computed following the procedure described in [89]. The phasic features were calculated within a time window (response window) $5 \mathrm{~s}$ long after the stimulus onset. After each unpredictable lateral strong skids was extracted, the number of SCRs within a response window (nSCR), the latency of first SCR (Lat), the amplitude-sum of SCRs (reconvolved from phasic driver peaks; AmpSum), the average phasic driver activity (Mean.SCR; time integral over response window by size of response window), the variance of the phasic driver signal (Var.SCR), the phasic driver area under curve (AUC.SCR), the max phasic driver amplitude (Max.SCR). From the tonic driver signal were extracted features as the average level of (decomposed) tonic component (Mean.Tonic), the variance of the tonic driver signal (Var.Tonic), and the number of the nonspecific response (i.e., the spontaneous SCR unrelated to a specific stimulus; NSR). Table I reports all of the mechanical and physiological features. 


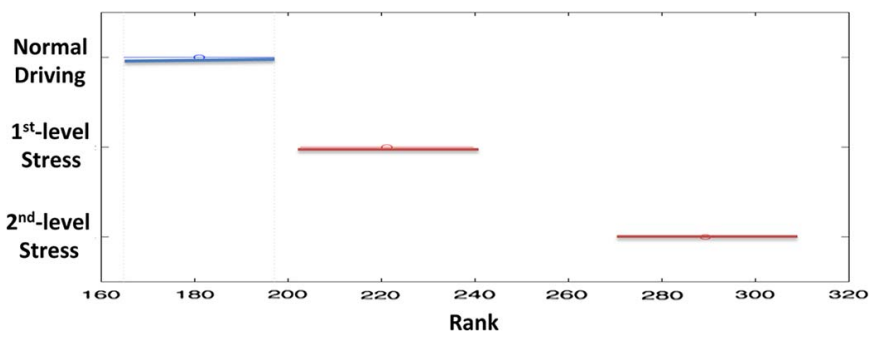

Fig. 5. Statistical comparison of means of SC tonic among normal and stressed driving sessions.

\section{E. Pattern Recognition}

A methodological approach based on machine learning has been applied to the features extracted from the acquired signals in order to distinguish among normal driving and first- and second-level stress sessions. More specifically, two different strategies have been adopted. First, we used all the mechanical and physiological features; then, we reduced the data set using a feature selection strategy based on uncorrelated statistical significant features. Finally, as the SC is generally considered strongly stimulus dependent, and being the only one that can be calculated in the short time window and time intervals between skids, we used only the SC features to discriminate the sessions. In order to choose the best pattern recognition methodology, we compared three different classifiers: nearest mean classifier (NMC), multilayer perceptron (MLP), and k-nearest neighbor $(\mathrm{k}-\mathrm{NN})$. Each classifier is representative of a specific pattern recognition approach, i.e., NMC is based on a template matching approach, MLP is based on a decision boundary construction approach, and finally, $\mathrm{k}-\mathrm{NN}$ is based on a probabilistic approach [90]. The NMC classifier showed the highest accuracy. For the sake of brevity and clarity, we reported here only the description and results relative to the NMC classifier.

1) NMC: NMC is a linear classification method based on an analogous approach to the well-known nearest neighbor rule (NN-rule). In the NN-rule, after the training phase of the classifier, test data are classified in the class of the nearest training sample, in terms of Euclidean distance, in feature space. In the NMC, a new sample of the test set is recognized to belong to that class whose mean has the minimum Euclidean distance from the class itself. More specifically, considering two classes, i.e., $w_{1}$ and $w_{2}$, NMC assigns unknown pattern $x$ (where $x \in \mathbb{R}_{d}, d$ is the dimension of the feature space), which is a realization of a random vector $X$, to that class whose mean is the nearest to the pattern $x$. The NMC discriminant function for the two-class problem can be written as

$$
g(x)=\left\|x-\bar{x}^{(2)}\right\|-\left\|x-\bar{x}^{(1)}\right\|+\ln \frac{P\left(w_{1}\right)}{P\left(w_{2}\right)}
$$

where $\bar{x}^{(1)}$ and $\bar{x}^{(2)}$ are sample means of training sets $L_{1}$ and $L_{2}$, and $P\left(w_{1}\right)$ and $P\left(w_{2}\right)$ are the prior probabilities of classes $w_{1}$ and $w_{2}$, respectively. Since squared Euclidean distance $\| x-$ $y \|=(x-y)(x-y)^{T}$ for $P\left(\omega_{1}\right)=P\left(\omega_{2}\right)=1 / 2$, the NMC can be rewritten in its standard form as

$$
g(x)=\left(\bar{x}^{(1)}-\bar{x}^{(2)}\right) x^{T}-\frac{1}{2}\left(\bar{x}^{(1)}-\bar{x}^{(2)}\right)\left(\bar{x}^{(1)}-\bar{x}^{(2)}\right)^{T} .
$$

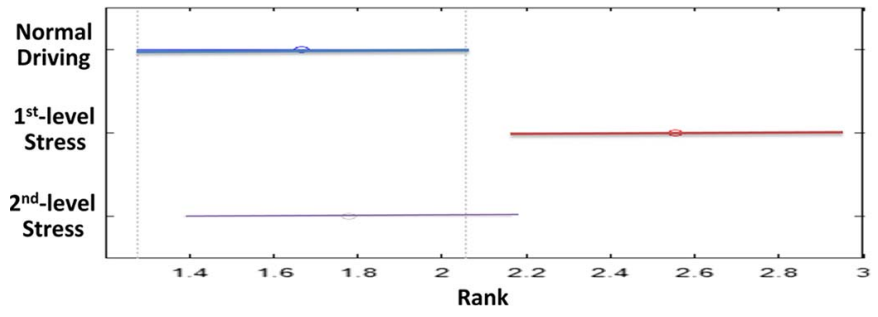

Fig. 6. Statistical comparison of LF/HF power of HRV among the three driving sessions with Bonferroni correction.

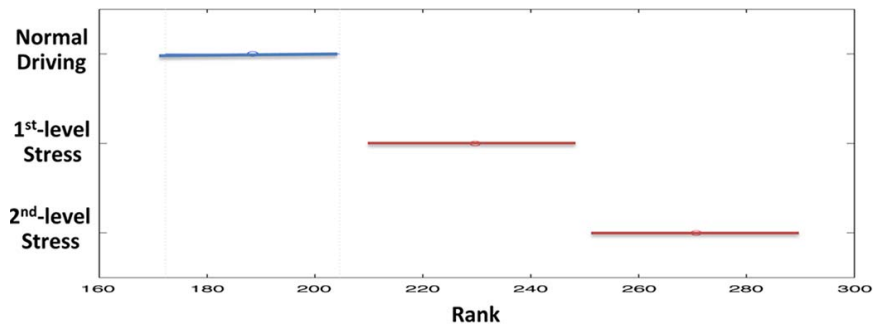

Fig. 7. Statistical comparison of means of SC phasic response among normal and stressed driving sessions.

The decision boundary of the NMC classifier is a hyperplane that is a perpendicular bisector of the line between the sample means of classes $\omega_{1}$ and $\omega_{2}$. After the training process, the performance of the classification task was evaluated using the confusion matrix. The generic element $r_{i j}$ of the confusion matrix indicates how many times in percentage a pattern belonging to class $i$ was classified as belonging to class $j$. A more diagonal confusion matrix corresponds to a higher degree of classification. We performed a leave-one-subject-out crossvalidation procedure [91]. More specifically, we performed a 15 -fold cross-validation where for each fold, the training set was comprised of data coming from 14 subjects, and the test set was comprised of the data coming from the remaining subject; this procedure was iteratively repeated for all the subjects. At the end of this process, results are summarized as mean and standard deviation among the 15 confusion matrices.

\section{EXPERIMENTAL RESULTS}

Concerning the psychological evaluation results, the STAI-Y1 scores increased from $33.74 \pm 3$ before starting the task to $38.58 \pm 8$ after the task ( $p<0.05$, t-test for paired samples). This result shows a significant increase in anxiety/stress level induced by the task. None of the volunteers reported a STAI-Y2 score above the cutoff normative threshold for the scale as provided by the Italian version of the scale [92]. The mechanical and physiological features calculated in the small transient after the lateral skid, as well as features computed in the time intervals between consecutive lateral skid stimuli, were considered in the analysis. For both of them, an intersubject statistical analysis was carried out to test the null hypothesis of having no statistical difference among the three driving sessions (i.e., normal driving, first-level stress session, and second-level stress session) by using a nonparametric test, i.e., the Friedman test with the Bonferroni post-hoc correction 
This article has been accepted for inclusion in a future issue of this journal. Content is final as presented, with the exception of pagination.

TABLE II

LIST OF THE UNCORRELATED STATISTICALLY SigNificANT FEATURES $(p<0.05)$

\begin{tabular}{c|c|c}
\hline Source & Parameter & $\mathbf{p}$-value \\
\hline \hline$H R V$ & Mean of the R-R intervals & $\mathrm{p}<0.05$ \\
\hline$H R V$ & peak-frequency in the HF band & $\mathrm{p}<0.05$ \\
\hline$R S P$ & Respiratory rate & $\mathrm{p}<0.02$ \\
\hline$R S P$ & Power within the band $0.1-0.2 \mathrm{~Hz}$ & $\mathrm{p}<0.02$ \\
\hline$R S P$ & Skewness & $\mathrm{p}<0.05$ \\
\hline Tonic EDR & Mean value & $\mathrm{p}<0.001$ \\
\hline Tonic EDR & Number of NSR & $\mathrm{p}<0.05$ \\
\hline Tonic EDR & Variance & $\mathrm{p}<0.02$ \\
\hline Phasic EDR & Number of SCR & $\mathrm{p}<0.05$ \\
\hline Phasic EDR & Variance & $\mathrm{p}<0.01$ \\
\hline Phasic EDR & Peak amplitude & $\mathrm{p}<0.02$ \\
\hline Phasic EDR & Average phasic driver activity & $\mathrm{p}<0.03$ \\
\hline Phasic $E D R$ & Latency & $\mathrm{p}<0.03$ \\
\hline Phasic EDR & Area Under Curve & $\mathrm{p}<0.01$ \\
\hline Vehicle & Steering Wheel Angle Correction & $\mathrm{p}<0.05$ \\
\hline Vehicle & Vehicle Velocity Difference & $\mathrm{p}<0.05$ \\
\hline P-vales $w e$
\end{tabular}

P-values were obtained from the Freedman non-parametric test for statistical differences with Bonferroni correction.

[93]. Each feature was compared by averaging the response of each subject to the different stimuli in the same session and applying the test to the obtained sample data. Concerning the HRV analysis, all the features resulted to be statistically different for at least one session out of three. As an example, statistical comparison of LF/HF power of HRV among the three driving sessions with Bonferroni correction is reported in Fig. 6. Analogously, the respiratory signal showed a significant difference in the respiratory rate, power within the band $0.1-0.2 \mathrm{~Hz}$, and skewness features in at least one session. Finally, all of the features of the SC phasic components, relative to the transients after skid, as well as SC tonic components computed during steady driving were able to discriminate all of the three driving sessions. As an example, Figs. 5-7 show an increase of the sympathetic activity as the stressing level grows. Moreover, many details on the sympathetic contribution of EDR features can be found in [17]. Concerning the vehicle's parameters, we found a statistical difference between the stressful driving sessions only for features extracted in time windows between the skids that started after $5 \mathrm{~s}$ of the predefined response window [25], i.e., speed and steering angle differences from the ideal target profile. In Table II, the list of the uncorrelated statistically significant features is reported. Given all of the physiological parameters, we discriminated the three driving sessions by evaluating the three following feature sets:

- $\alpha$ : all the features coming from the HRV, RSP, EDR (tonic and phasic), and vehicle parameters;

- $\beta$ : all the statistically significant features among the HRV, RSP, EDR (tonic and phasic), and vehicle parameters;

- $\gamma$ : all the EDR features (tonic and phasic).

In Table III, the complete feature set was used to an automatic recognition of the three driving sessions by means of an NMC classifier. Table IV shows results when only features with significant differences among the three driving sessions are used as input to the NMC classifier. Looking at the state of the art and in view of future applications, the SCR seems to be the most suitable biosignal for the stressed driving assessment. In addition, such a signal could be acquired by means of a sen-
TABLE III

Confusion Matrix of THE NMC FOR THE THREe-Class Problem With All of the Mechanical and Physiological Features

\begin{tabular}{|c|c|c|c|}
\hline MNC & $\begin{array}{c}\text { Normal } \\
\text { Driving }\end{array}$ & $\begin{array}{c}1^{\text {st }} \text {-level } \\
\text { Stress }\end{array}$ & $\begin{array}{c}2^{\text {nd }} \text {-level } \\
\text { Stress }\end{array}$ \\
\hline \hline Normal Driving & $\begin{array}{c}\mathbf{9 5 . 3 3} \pm \mathbf{8 . 3 3} \\
(7292)\end{array}$ & $\begin{array}{c}5.92 \pm 11.78 \\
(451)\end{array}$ & $\begin{array}{c}1.48 \pm 3.90 \\
(113)\end{array}$ \\
\hline $1^{\text {st }}$-level Stress & $\begin{array}{c}3.33 \pm 7.23 \\
(229)\end{array}$ & $\begin{array}{c}\mathbf{8 2 . 2 2} \pm \mathbf{2 0 . 4 8} \\
(5659)\end{array}$ & $\begin{array}{c}3.70 \pm 11.63 \\
(254)\end{array}$ \\
\hline $2^{\text {nd } \text {-level Stress }}$ & $\begin{array}{c}1.33 \pm 5.16 \\
(90)\end{array}$ & $\begin{array}{c}11.85 \pm 14.82 \\
(779)\end{array}$ & $\begin{array}{c}\mathbf{9 4 . 8 0} \pm \mathbf{1 1 . 7 8} \\
(6527)\end{array}$ \\
\hline
\end{tabular}

Results were obtained after 15 LOSO cross validation

TABLE IV

CONFUSION MATRIX OF THE NMC FOR THE THREE-ClasS Problem With All of the Significant Features

\begin{tabular}{|c|c|c|c|}
\hline MNC & $\begin{array}{c}\text { Normal } \\
\text { Driving }\end{array}$ & $\begin{array}{c}1^{\text {st }} \text {-level } \\
\text { Stress }\end{array}$ & $\begin{array}{c}2^{\text {nd }} \text {-level } \\
\text { Stress }\end{array}$ \\
\hline \hline Normal Driving & $\begin{array}{c}\mathbf{9 6 . 0 0} \pm \mathbf{5 . 6 0} \\
(4032)\end{array}$ & $\begin{array}{c}7.04 \pm 21.27 \\
(294)\end{array}$ & $\begin{array}{c}2.23 \pm 8.60 \\
(93)\end{array}$ \\
\hline $1^{s t}$-level Stress & $\begin{array}{c}1.34 \pm 5.16 \\
(50)\end{array}$ & $\begin{array}{c}\mathbf{9 1 . 1 1} \pm \mathbf{2 3 . 8 3} \\
(3444)\end{array}$ & $\begin{array}{c}1.49 \pm 3.90 \\
(56)\end{array}$ \\
\hline $2^{\text {nd } \text {-level Stress }}$ & $\begin{array}{c}2.66 \pm 2.68 \\
(100)\end{array}$ & $\begin{array}{c}1.48 \pm 3.91 \\
(56)\end{array}$ & $\begin{array}{c}\mathbf{9 5 . 2 8} \pm \mathbf{9 . 0 7} \\
(3602)\end{array}$ \\
\hline
\end{tabular}

Results were obtained after 15 LOSO cross validation

TABLE V

CONFUSION MATRIX OF THE NMC FOR THE THREE-ClasS Problem With All of the EDR Features

\begin{tabular}{|c|c|c|c|}
\hline MNC & $\begin{array}{c}\text { Normal } \\
\text { Driving }\end{array}$ & $\begin{array}{c}1^{s t} \text {-level } \\
\text { Stress }\end{array}$ & $\begin{array}{c}2^{n d} \text {-level } \\
\text { Stress }\end{array}$ \\
\hline \hline Normal Driving & $\mathbf{9 0 . 0 0} \pm \mathbf{1 5 . 1 1}$ & $5.30 \pm 10.5$ & $0.34 \pm 1.83$ \\
& $(1485)$ & $(87)$ & $(6)$ \\
\hline $1^{s t}$-level Stress & $\begin{array}{c}6.89 \pm 7.58 \\
(102)\end{array}$ & $\begin{array}{c}\mathbf{8 9 . 6 3} \pm \mathbf{5 . 5 3} \\
(1131)\end{array}$ & $\begin{array}{c}0.40 \pm 1.03 \\
(6)\end{array}$ \\
\hline $2^{\text {nd } \text {-level Stress }}$ & $\begin{array}{c}3.11 \pm 8.32 \\
(46)\end{array}$ & $\begin{array}{c}5.07 \pm 13.17 \\
(75)\end{array}$ & $\begin{array}{c}\mathbf{9 9 . 2 3} \pm \mathbf{2 . 8 7} \\
(1474)\end{array}$ \\
\hline
\end{tabular}

Results were obtained after 15 LOSO cross validation

TABLE VI

Number OF THE EXTRACTED CASES

\begin{tabular}{|c|c|c|c|}
\hline & $\begin{array}{c}\text { Normal } \\
\text { Driving }\end{array}$ & $\begin{array}{c}1^{\text {st }} \text {-level } \\
\text { Stress }\end{array}$ & $\begin{array}{c}2^{\text {nd }} \text {-level } \\
\text { Stress }\end{array}$ \\
\hline \hline Physiological \& Mechanical & 7650 & 6885 & 6885 \\
\hline Statistically Significant & 4200 & 3780 & 3780 \\
\hline EDR & 1650 & 1485 & 1485 \\
\hline
\end{tabular}

sorized glove (see, e.g., [23]) or via ad hoc electrodes integrated in the steering wheel, for instance. Therefore, the data set $\gamma$ was constituted by all of the features coming only from the tonic and phasic SCR. The results in terms of the confusion matrix for the three-class problems are reported in Table V. Moreover, Table VI reports the number of the extracted cases along the three driving conditions per mechanical and physiological signals, only statistically significant features and features extracted only from the electrodermal response. Fig. 8 shows the overall accuracy computed per subject for the three considered feature sets, i.e., physiological and mechanical, statistically significant, and EDR, respectively. Moreover, Table VII shows the sensitivity, specificity, and accuracy of the classification over the three data sets. Specifically, sensitivity, and specificity have been computed for each class and are intended as true positive rate 
This article has been accepted for inclusion in a future issue of this journal. Content is final as presented, with the exception of pagination.

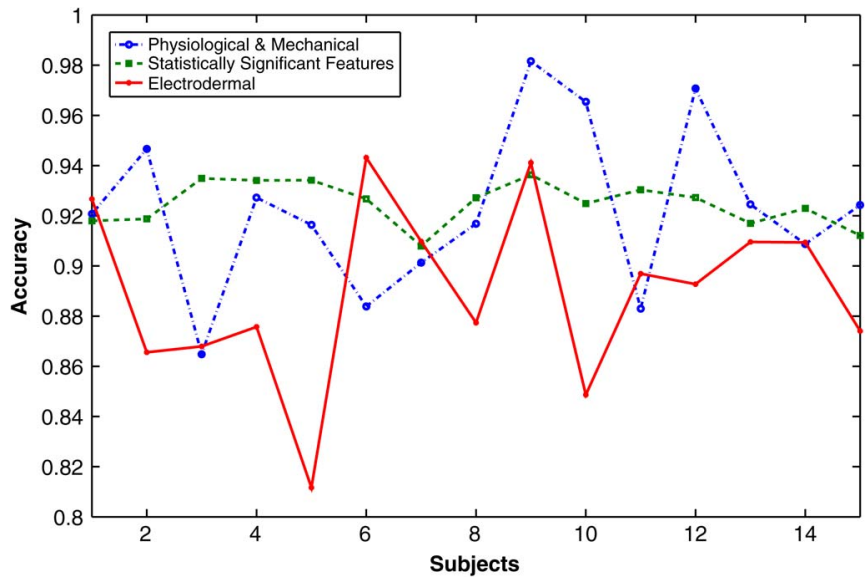

Fig. 8. Overall accuracy computed per subject for the three sets of features.

TABLE VII

Sensitivity, SPECIFICITy, AND ACCURACy OF THE THREe-Class Recognition Process Over the Three Different Data Sets

\begin{tabular}{|c|c|c|c|c|}
\hline & & $\begin{array}{c}\text { Physiological } \\
\text { and } \\
\text { Mechanical }\end{array}$ & $\begin{array}{c}\text { Statistically } \\
\text { significant } \\
\text { features }\end{array}$ & EDR \\
\hline Normal & Sensitivity & 0.94 & 0.96 & 0.92 \\
Driving & Specificity & 0.96 & 0.97 & 0.96 \\
& Accuracy & 0.92 & 0.92 & 0.9 \\
\hline $1^{\text {st }}$ & Sensitivity & 0.83 & 0.86 & 0.86 \\
level & Specificity & 0.95 & 0.95 & 0.93 \\
Stress & Accuracy & 0.9 & 0.91 & 0.87 \\
\hline $2^{\text {nd }}$ & Sensitivity & 0.95 & 0.95 & 0.92 \\
level & Specificity & 0.95 & 0.96 & 0.96 \\
Stress & Accuracy & 0.9 & 0.91 & 0.91 \\
\hline & Overall Accuracy & $\mathbf{0 . 9 1}$ & $\mathbf{0 . 9 2}$ & $\mathbf{0 . 9}$ \\
\hline
\end{tabular}

and true negative rate for that class, respectively. Accuracy is calculated as the correct classifications of one class divided by the number of classifications related to that class. In addition, we also computed the overall accuracy, which is the overall correctness of the model and corresponds to the sum of correct classifications divided by the total number of classifications.

\section{CONCLUSION AND Discussions}

This study reports on how physiological signs (from human subjects) as well as mechanical parameters (from the vehicle) change when the level of stress increases during a simulated driving. Fifteen healthy subjects volunteered to participate in the experiment where one normal driving and two stress-level conditions are simulated. The first level of stress is induced by applying unpredictable skids to the vehicle, whereas the second increased level of stress is obtained by administering to the subjects a series of time-pressing mathematical questions, in addition to the previous unpredictable skids. The hypothesis behind the experiments is that when an incremental stress is induced by external conditions, by means of mental and mechanical stimuli, subjects modify their sympathovagal balance resulting in a change in driving performance with respect to a normal driving style. Experiments were performed by using a driving simulator, guaranteeing a more effective and reliable experimental setup [63]. Standard features were extracted in order to constitute an input data set for an effective data- mining procedure (based on an NMC classifier model). Three feature sets, namely, $\alpha, \beta$, and $\gamma$, were composed by taking into account all the features, all the statistical significant features (see Table III), and all the EDR features, respectively. For each data set, a three-class problem to discriminate the three driving sessions was considered. The best result is obtained with the data set $\beta$, i.e., considering all of the statistical significant and uncorrelated parameters, with accuracy greater than $90 \%$.

The discrimination results among the three driving sessions are very promising even when only the EDR features (data set $\gamma$ ) were taken into account. Our goal was to study the human-machine interface when an increasing level of stress is induced during a simulated driving. Psychological evaluation revealed that the experimental task was able to significantly increase the levels of subjective anxiety and stress in the volunteers as measured by STAI-Y1. STAI-Y1 and subjective evaluation of stress and anxiety levels are currently widely used to evaluate the efficacy of stressful stimulating protocols. The significant increase of the scores after the task allows us to sustain that the experimental design induced stress. Moreover, starting from an initial condition where unpredictable skids can occur, additional mathematical questions were asked to the subjects in order to further engage their mental resources and increase the psychophysiological load. Comparing the two configurations, we found that during the small transient after the skid, the sympathovagal balance of the subjects is modified in terms of the EDR phasic component, whereas the mechanical parameters of the vehicle remain statistically unchanged. During the time intervals between skids, in addition to the change in the physiological signs of the subjects, we observed also a statistical change in the vehicle behavior. Moreover, we exploited these statistical findings, implementing a machine learning method, based on an NMC classifier, showing accuracy greater than $90 \%$ when both mechanical and physiological features are used. Finally, the EDR being the only signal that can be studied during the transient as well as in the steady driving and it being very sensitive to stimulus changes, we used only the EDR features in the classifier, achieving satisfactory and acceptable results. Accordingly, in the future, EDR signal could be used as an indirect stress marker for real driving applications. Real driving could benefit from the proposed approach in order to detect incipient growing levels of stress and alert the driver or activate safety measures of the vehicle, thus reducing the risk of accidents or collisions. For example, a pair of electrodes could be embedded into the steering wheel to acquire the EDR, and systems based on ultrawideband [94], [95] could be integrated into the seat to acquire ECG and RSP. Alternatively, wearable monitoring systems could be directly worn by the driver, although this latter solution could be uneasy and awkward. The outcomes of this study show that by monitoring the changes in the ANS, it is possible to indirectly evaluate the driver stress level. Moreover, studies on integrated information among physiological, psychological, and cognitive, by mathematical models, for estimating driver workload revealed that increasing the number of physiological inputs improves the model performance [96], [97]. Naturalistic studies have shown how increased driver workload is often due to the incoming information from novel car systems that, even if provide 
helpful information, sometime produce stressing overlapping phenomena that alter the driver status [98]. Results of these studies could be integrated into a new generation of integrated workload management driver assistance systems, allowing, e.g., a novel implementation of intelligent engines, where the physiological and psychological driver status as well as the vehicle status could be combined with information coming from the external environment, such as traffic, weather, accidents, and so on [99], [100]. In this view, these systems could play a relevant role in driver safety control and maintenance.

\section{REFERENCES}

[1] H. Selye, "A syndrome produced by diverse nocuous agents," Nature, vol. 138, p. 32, 1936

[2] A. Stokes and K. Kite, "On grasping a nettle and becoming emotional," in Stress, Workload, and Fatigue. Mahwah, NJ, USA: Lawrence Erlbaum, 2001, pp. 162-181.

[3] P. Broadhurst, "Emotionality and the Yerkes-Dodson law," J. Exp. Psychol., vol. 54, no. 5, pp. 345-352, Nov. 1957.

[4] R. Lazarus, Emotion and Adaptation. New York, NY, USA: Oxford Univ. Press, 1991.

[5] G. Valenza et al., "Wearable monitoring for mood recognition in bipolar disorder based on history-dependent long-term heart rate variability analysis," IEEE J. Biomed. Health Informat., vol. 18, no. 5, pp. 1625-1635, Sep. 2014.

[6] J. Panksepp, "Affective neuroscience: A paradigm to study the animate circuits for human emotions," in Emotion: Interdisciplinary Perspectives. Mahwah, NJ, USA: Lawrence Erlbaum, 1996, pp. 29-60.

[7] A. Lanata, G. Valenza, and E. P. Scilingo, "Eye gaze patterns in emotional pictures," J. Ambient Intell. Human. Comput., vol. 4, no. 6, pp. 705-715, 2013.

[8] N. Karim, J. Hasan, and S. Ali, "Heart rate variability-A review," J. Basic Appl. Sci., vol. 7, no. 1, pp. 71-77, 2011.

[9] G. Valenza, L. Citi, A. Lanatà, E. P. Scilingo, and R. Barbieri, "A nonlinear heartbeat dynamics model approach for personalized emotion recognition," in Proc. Annu. Int. Conf. IEEE EMBS, 2013, pp. 2579-2582.

[10] U. Rajendra Acharya, K. Paul Joseph, N. Kannathal, C. M. Lim, and J. S. Suri, "Heart rate variability: A review," Med. Biol. Eng. Comput., vol. 44, no. 12, pp. 1031-1051, 2006.

[11] G. Valenza, P. Allegrini, A. Lanatà, and E. P. Scilingo, "Dominant Lyapunov exponent and approximate entropy in heart rate variability during emotional visual elicitation," Front. Neuroeng., vol. 5, no. 3, p. 7, 2012.

[12] G. Valenza, L. Citi, A. Lanatà, E. P. Scilingo, and R. Barbieri, "Revealing real-time emotional responses: A personalized assessment based on heartbeat dynamics," Sci. Rep., vol. 4, no. 4998, pp. 1-13, 2014.

[13] G. Valenza, L. Citi, and R. Barbieri, "Instantaneous nonlinear assessment of complex cardiovascular dynamics by Laguerre-Volterra point process models," in Proc. IEEE-EMBC, 2013, pp. 6131-6134.

[14] A. Lanata et al., "Comparative evaluation of susceptibility to motion artifact in different wearable systems for monitoring respiratory rate," IEEE Trans. Inf. Technol. Biomed., vol. 14, no. 2, pp. 378-386, Mar. 2010.

[15] G. Valenza, A. Lanata, and E. P. Scilingo, "Oscillations of heart rate and respiration synchronize during affective visual stimulation," IEEE Trans. Inf. Technol. Biomed., vol. 16, no. 4, pp. 683-690, Jul. 2012.

[16] G. Valenza, A. Lanata, and E. P. Scilingo, "Improving emotion recognition systems by embedding cardiorespiratory coupling," Physiol. Meas., vol. 34, no. 4, pp. 449-464, Apr. 2013.

[17] W. Boucsein, Electrodermal Activity. New York, NY, USA: SpringerVerlag, 2011

[18] A. Greco et al., "On the deconvolution analysis of electrodermal activity in bipolar patients," in Proc. Int. Conf. IEEE , 2012, pp. 6691-6694.

[19] E. P. Scilingo, A. Lanata, and A. Tognetti, Sensors for Wearable Systems. New York, NY, USA: Springer-Verlag, 2011.

[20] G. Valenza, A. Lanata, M. Ferro, and E. P. Scilingo, "Real-time discrimination of multiple cardiac arrhythmias for wearable systems based on neural networks," in Proc. Comput. Cardiol., 2008, pp. 1053-1056.

[21] E. Scilingo et al., "Performance evaluation of sensing fabrics for monitoring physiological and biomechanical variables," IEEE Trans. Inf. Technol. Biomed., vol. 9, no. 3, pp. 345-352, Sep. 2005.
[22] G. Valenza, C. Gentili, A. Lanata, and E. P. Scilingo, "Mood recognition in bipolar patients through the psyche platform: Preliminary evaluations and perspectives," Artif. Intell. Med., vol. 57, no. 1, pp. 49-58, Jan. 2013.

[23] A. Lanata, G. Valenza, and E. Scilingo, "A novel EDA glove based on textile-integrated electrodes for affective computing," Med. Biol. Eng. Comput., vol. 50, no. 11, pp. 1163-1172, Nov. 2012

[24] G. Valenza, A. Lanata, E. P. Scilingo, and D. De Rossi, "Towards a smart glove: Arousal recognition based on textile electrodermal response," in Proc. Int. Conf. IEEE Eng. Med. Biol. Soc., 2010, pp. 3598-3601.

[25] R. Bartolozzi and F. Frendo, "Evaluation of drivers' attention level by driving simulator," in Proc. ASME Conf., 2012, pp. 155-165.

[26] M. Helander, "Applicability of drivers' electrodermal response to the design of the traffic environment," J. Appl. Psychol., vol. 63, no. 4, pp. 481-488, Aug. 1978.

[27] P. Feldman, S. Cohen, N. Hamrick, and S. Lepore, "Psychological stress, appraisal, emotion and cardiovascular response in a public speaking task," Psychol. Health, vol. 19, no. 3, pp. 353-368, Jun. 2004.

[28] E. Duffy, "The conceptual categories of psychology; A suggestion for revision," Psychol. Rev., vol. 48, no. 3, pp. 177-203, May 1941.

[29] C. D. Wickens, "Processing resources and attention," in Multiple-Task Performance. Bristol, PA, USA: Taylor \& Francis, 1991, pp. 3-34.

[30] M. Inderbitzin et al., "The social perceptual salience effect," $J$. Exp. Psychol. Hum. Percept. Perform., vol. 39, no. 1, pp. 62-74, Feb. 2013.

[31] A. Murata, "Foveal task complexity and visual funneling," Hum. Factors, J. Hum. Factors Ergonom. Soc., vol. 46, no. 1, pp. 135-141, 2004.

[32] R. Backs, "An autonomic space approach to the psychophysiological assessment of mental workload," in Stress, Workload, and Fatigue. Human Factors in Transportation. Mahwah, NJ, USA: Lawrence Erlbaum, 2001, pp. 279-289.

[33] J. Cacioppo and L. Tassinary, "Inferring psychological significance from physiological signals," Amer. Psychol., vol. 45, no. 1, pp. 16-28, Jan. 1990.

[34] P. Hancock, N. Meshkati, and M. Robertson, "Physiological reflections of mental workload," Aviat., Space, Environ. Med., vol. 56, no. 11, pp. 1110-1114, Nov. 1985.

[35] T. Suzuki, Y. Nakamura, and T. Ogasawara, "Intrinsic properties of driver attentiveness," Expressway Automob., vol. 9, pp. 24-29, 1966.

[36] T. Rockwell, "Spare visual capacity in driving-revisited: New empirical results for an old idea," in Proc. 2nd Int. Conf. Vis. Veh., 1988, pp. 317-324.

[37] R. Fernandez and R. Picard, "Modeling drivers speech under stress," Speech Commun., vol. 40, no. 1/2, pp. 145-159, Apr. 2003.

[38] J. Healey and R. Picard, "Detecting stress during real-world driving tasks using physiological sensors," IEEE Trans. Intell. Transp. Syst., vol. 6, no. 2, pp. 156-166, Jun. 2005.

[39] R. Allen, H. R. Jex, D. T. McRuer, and R. J. Dimarco, "Alcohol effects on driving behavior and performance in a car simulator," IEEE Trans. Syst., Man, Cybern., vol. SMC-5, no. 5, pp. 498-505, Sep. 1975.

[40] A. Desai et al., "The utility of the AusEd driving simulator in the clinical assessment of driver fatigue," Behav. Res. Methods, vol. 39, no. 3, pp. 673-681, Aug. 2007.

[41] H. Alm and L. Nilsson, "Changes in driver behaviour as a function of handsfree mobile phones-A simulator study," Accid. Anal. Prevent., vol. 26, no. 4, pp. 441-451, Aug. 1994.

[42] G. Matthews, T. Sparkes, and H. Bygrave, "Attentional overload, stress, and simulate driving performance," Hum. Perform., vol. 9, no. 1, pp. 77101, 1996.

[43] R. Koppa, Human Factors, Traffic Flow Theory-A State-of-The-Art Report.

[44] P. Ting, J. Hwang, J. Doong, and M. Jeng, "Driver fatigue and highway driving: A simulator study," Physiol. Behav., vol. 94, no. 3, pp. 448-453, Jun. 2008.

[45] R. E. Dewar and P. L. Olson, Human Factors in Traffic Safety. Tucson, AZ, USA: Lawyers \& Judges Pulishing Company, Inc., 2007.

[46] F. Barbe et al., "Automobile accidents in patients with sleep apnea syndrome: An epidemiological and mechanistic study," Amer. J. Respiratory Crit. Care Med., vol. 158, no. 1, pp. 18-22, 1998.

[47] R. Bartolozzi and F. Frendo, "Definition of simulated driving tests for the evaluation of drivers reactions and responses," Traffic Injury Prevent., vol. 15 , no. 3, pp. 302-309, 2014.

[48] W. Wierwille, J. Casali, and B. Repa, "Driver steering reaction time to abrupt-onset crosswinds, as measured in a moving-base driving simulator," Hum. Factors, J. Hum. Factors Ergonom. Soc., vol. 25, no. 1, pp. 103-116, 1983 . 
[49] S. Banks, P. Catcheside, L. Lack, R. R. Grunstein, and R. D. McEvoy, "Low levels of alcohol impair driving simulator performance and reduce perception of crash risk in partially sleep deprived subjects," Sleep, vol. 27, no. 6, pp. 1063-1067, Sep. 2004.

[50] A. Riener, A. Ferscha, and M. Aly, "Heart on the road: HRV analysis for monitoring a driver's affective state," in Proc. 1st Int. Conf. Autom. User Interfaces Interact. Veh. Appl., 2009, pp. 99-106.

[51] N. Hjortskov et al., "The effect of mental stress on heart rate variability and blood pressure during computer work," Eur. J. Appl. Physiol., vol. 92, no. 1, pp. 84-89, Jun. 2004.

[52] H. Cohen et al., "Power spectral analysis of heart rate variability in posttraumatic stress disorder patients," Biol. Psychiatry, vol. 41, no. 5, pp. 627-629, Mar. 1997.

[53] W. Langewitz, H. Rüddel, and H. Schächinger, "Reduced parasympathetic cardiac control in patients with hypertension at rest and under mental stress," Amer. Heart J., vol. 127, no. 1, pp. 122-128, Jan. 1994.

[54] J. Zhai and A. Barreto, "Stress detection in computer users based on digital signal processing of noninvasive physiological variables," in Proc. 28th Annu. Int. Conf. IEEE EMBS, 2006, pp. 1355-1358.

[55] E. Jovanov et al., "Stress monitoring using a distributed wireless intelligent sensor system," IEEE Eng. Med. Biol. Mag., vol. 22, no. 3, pp. 49-55, May/Jun. 2003.

[56] R. Lazarus, J. Speisman, and A. Mordkoff, "The relationship between autonomic indicators of psychological stress: Heart rate and skin conductance," Psychosom. Med., vol. 25, no. 1, pp. 19-30, Jan. 1963.

[57] A. de Santos Sierra, C. S. Avila, J. Guerra Casanova, and G. B. del Pozo, "A stress detection system based on physiological signals and fuzzy logic," IEEE Trans. Ind. Electron., vol. 58, no. 10, pp. 4857-4865, Oct. 2011.

[58] J. O'Hanlon, Heart Rate Variability: A New Index of Driver Alertness/ Fatigue. Detroit, MI, USA: Soc. Autom. Eng.

[59] S. Conjeti, R. R. Singh, and R. Banerjee, "Bio-inspired wearable computing architecture and physiological signal processing for on-road stress monitoring," in Proc. IEEE-EMBS Int. Conf. BHI, 2012, pp. 479-482.

[60] C. Katsis, Y. Goletsis, G. Rigas, and D. Fotiadis, "A wearable system for the affective monitoring of car racing drivers during simulated conditions," Transp. Res. C, Emerg. Technol., vol. 19, no. 3, pp. 541-551, Jun. 2011.

[61] C. D. Spielberger and S. J. Sydeman, State-trait anxiety inventory and state-trait anger expression inventory. Hillsdale, NJ, USA: Lawrence Erlbaum, 1994

[62] J. Wang et al., "Perfusion functional MRI reveals cerebral blood flow pattern under psychological stress," Proc. Nat. Acad. Sci. United States Amer, vol. 102, no. 49, pp. 17 804-17 809, 2005.

[63] P. Olson and R. Dewar, Human Factors in Traffic Safety. Tucson, AZ, USA: Lawyers \& Judges Publ. Comp., 2002.

[64] M. Losa, F. Frendo, A. Cofrancesco, and R. Bartolozzi, "A procedure for validating fixed-base driving simulators," Transport, vol. 28 , no. 4, pp. $420-430,2013$

[65] C. W. Quaedflieg, T. Meyer, and T. Smeets, "The imaging Maastricht acute stress test (iMAST): A neuroimaging compatible psychophysiological stressor," Psychophysiology, vol. 50, no. 8, pp. 758-766, Aug. 2013.

[66] K. Dedovic et al., "The Montreal imaging stress task: Using functional imaging to investigate the effects of perceiving and processing psychosocial stress in the human brain," J. Psychiatry Neurosci., vol. 30, no. 5, pp. 319-325, Sep. 2005.

[67] K. Petrowski, G.-B. Wintermann, and M. Siepmann, "Cortisol response to repeated psychosocial stress," Appl. Psychophysiol. Biofeedback, vol. 37, no. 2, pp. 103-107, Jun. 2012.

[68] A. Lanata, E. P. Scilingo, and D. De Rossi, "A multimodal transducer for cardiopulmonary activity monitoring in emergency," IEEE Trans. Inf. Technol. Biomed., vol. 14, no. 3, pp. 817-825, May 2010.

[69] A. Lanata, G. Valenza, C. Mancuso, and E. P. Scilingo, "Robust multiple cardiac arrhythmia detection through bispectrum analysis," Expert Syst. Appl., vol. 38, no. 6, pp. 6798-6804, Jun. 2011.

[70] L. Schamroth, An Introduction to Electrocardiography. Oxford, U.K.: Blackwell Sci. Publ., 1971.

[71] P. Stein, M. Bosner, R. Kleiger, and B. Conger, "Heart rate variability: A measure of cardiac autonomic tone," Amer. Heart J., vol. 127, no. 5, pp. 1376-1381, May 1994.

[72] B. Kohler, C. Hennig, and R. Orglmeister, "The principal of software QRS detection," IEEE Eng. Med. Biol., vol. 21, no. 1, pp. 42-57, Jan./Feb. 2002

[73] J. Pan and W. Tompkins, "A real-time QRS detection algorithm," IEEE Trans. Bio-Med. Eng., vol. BME-32, no. 3, pp. 230-236, Mar. 1985.
[74] N. Lippman, K. M. Stein, and B. B. Lerman, "Nonlinear predictive interpolation. A new method for the correction of ectopic beats for heart rate variability analysis," J. Electrocardiol., vol. 26, pp. 14-19, 1993.

[75] N. Lippman, K. M. Stein, and B. B. Lerman, "Comparison of methods for removal of ectopy in measurement of heart rate variability," Amer. J. Physiol.-Heart Circulatory Physiol., vol. 267, no. 1, pp. H411-H418, Jul. 1994.

[76] W. Winton, L. Putnam, and R. Krauss, "Facial and autonomic manifestations of the dimensional structure of emotion," J. Exp. Social Psychol., vol. 20, no. 3, pp. 195-216, May 1984.

[77] R. Edelberg, "Electrodermal mechanisms: A critique of the two-effector hypothesis and a proposed replacement," in Proc. Progr. Electrodermal Res. New York, NY, USA: Springer-Verlag, 1993, pp. 7-29.

[78] D. Fowles et al., "Publication recommendations for electrodermal measurements," Psychophysiology, vol. 18, no. 3, pp. 232-239, May 1981.

[79] P. Lang, M. Greenwald, M. Bradley, and A. Hamm, "Looking at pictures: Affective, facial, visceral, and behavioral reactions," Psychophysiology, vol. 30, no. 3, pp. 261-273, May 1993.

[80] P. Lang, "The emotion probe," American Psychologist, vol. 50, no. 5, pp. 372-385, May 1995.

[81] D. Levinson and R. Edelberg, "Scoring criteria for response latency and habituation in electrodermal research: A critique," Psychophysiology, vol. 22, no. 4, pp. 417-426, Jul. 1985.

[82] M. Benedek and C. Kaernbach, "A continuous measure of phasic electrodermal activity," J. Neurosci. Methods, vol. 190, no. 1, pp. 80-91, Jun. 2010.

[83] E. Garrett, "The Bateman function revisited: A critical reevaluation of the quantitative expressions to characterize concentrations in the one compartment body model as a function of time with first-order invasion and first-order elimination," J. Pharmacokinetics Pharmacodyn., vol. 22, no. 2, pp. 103-128, Apr. 1994.

[84] R. Schneider, "A mathematical model of human skin conductance," Psychophysiology, vol. 24, no. 5, p. 610, 1987.

[85] H. Akaike, "Fitting autoregressive models for prediction," Anna. Inst. Statist. Math., vol. 21, no. 1, pp. 243-247, 1969.

[86] M. Malik, "Heart rate variability: Standards of measurement, physiological interpretation, and clinical use," Circulation, vol. 93, no. 5, pp. $1043-$ 1065, Mar. 1996.

[87] S. Koelstra et al., "Single trial classification of EEG and peripheral physiological signals for recognition of emotions induced by music videos," in Proc. Brain Informat., 2010, pp. 89-100.

[88] S. Mitra, Digital Signal Processing. A Computer-Based Approach New York, NY, USA: McGraw-Hill, 2001.

[89] A. Greco, G. Valenza, A. Lanata, G. Rota, and E. P. Scilingo, "Electrodermal activity in bipolar patients during affective elicitation," IEEE J. Biomed. Health Informat., vol. 18, no. 6, pp. 1865-1873, Nov. 2014. doi:10.1109/HAPTICS.2014.6775522.

[90] A. Jain, R. Duin, and J. Mao, "Statistical pattern recognition: A review," IEEE Trans. Pattern Anal. Mach. Intell., vol. 22, no. 1, pp. 4-37, Jan. 2000.

[91] M. Esterman, B. J. Tamber-Rosenau, Y.-C. Chiu, and S. Yantis, "Avoiding non-independence in fMRI data analysis: Leave one subject out," Neuroimage, vol. 50, no. 2, pp. 572-576, Apr. 2010.

[92] L. Pedrabissi and M. Santinello, Inventario per lansia di Stato e di Tratto: Nuova Versione Italiana dello STAI Forma Y: Manuale. Firenze, Italy: Organizzazioni Speciali.

[93] M. Friedman and A. J. Schwartz, "Alternative approaches to analyzing economic data," Amer. Econom. Rev., vol. 18, no. 1, pp. 39-49, Mar. 1991.

[94] D. Zito et al., "Feasibility study and design of a wearable system-ona-chip pulse radar for contactless cardiopulmonary monitoring," Int. J. Telemed. Appl., vol. 2008, 2008, Art. ID. 328597.

[95] D. Zito et al., "Wearable system-on-a-chip UWB radar for health care and its application to the safety improvement of emergency operators," in Proc. IEEE Int. Conf. Eng. Med. Biol. Soc., 2007, pp. 2651-2654.

[96] P. Besson et al., "Effectiveness of physiological and psychological features to estimate helicopter pilots' workload: A Bayesian network approach," IEEE Trans. Intell. Transp. Syst., vol. 14, no. 4, pp. 1872-1881, Dec. 2013.

[97] B. Lin and C. Wu, "Mathematical modeling of the human cognitive system in two serial processing stages with its applications in adaptive workload-management systems," IEEE Trans. Intell. Transp. Syst., vol. 12, no. 1, pp. 221-231, Mar. 2011.

[98] C. Wu, O. Tsimhoni, and Y. Liu, "Development of an adaptive workload management system using the Queueing Network-Model Human 
Processor (QN-MHP)," IEEE Trans. Intell. Transp. Syst., vol. 9, no. 3, pp. 463-475, Sep. 2008.

[99] G. Rigas, Y. Goletsis, and D. I. Fotiadis, "Real-time driver's stress event detection," IEEE Trans. Intell. Transp. Syst., vol. 13, no. 1, pp. 221-234, Mar. 2012.

[100] N. Saunier, T. Sayed, and C. Lim, "Probabilistic collision prediction for vision-based automated road safety analysis," in Proc. IEEE ITSC, 2007, pp. 872-878.

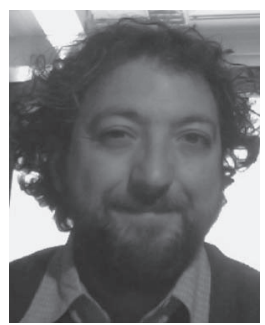

Antonio Lanatà (M'12) received the Laurea degree in electronic engineering, with a dissertation on electroactive polymers for actuator systems, and the $\mathrm{Ph} . \mathrm{D}$. degree in automation, robotics, and bioengineering from University of Pisa, Pisa, Italy, in 2001 and 2006, respectively.

In 2007 he received a postdoctoral fellowship on innovative cardiopulmonary systems monitoring. He is the author of numerous articles in international scientific journals and several chapters of books. His research interests are focused on developing wireless wearable systems, ultrasound and ultrawideband radar systems for vital signal monitoring, and biomedical signal processing for health status detection.

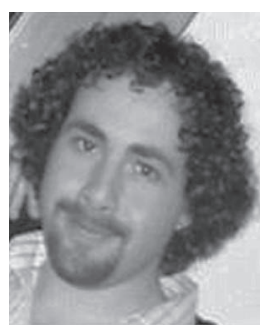

Gaetano Valenza (S'10-M'12) received the Ph.D. degree in automation, robotics, and bioengineering from University of Pisa, Pisa, Italy, in 2013.

He is a Postdoctoral Fellow with the Department of Information Engineering and the "E. Piaggio" Research Center, University of Pisa. His research is focused biomedical signal processing, cardiovascular and neural modeling, and wearable systems for physiological monitoring. Applicative fields include affective computing, assessment of mood disorders, and characterization of disorder of consciousness.

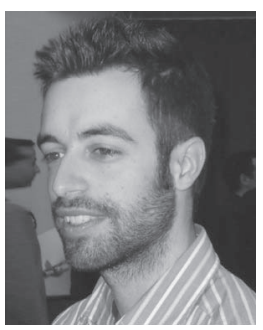

Alberto Greco received the Laurea degree in biomedical engineering from University of Pisa, Pisa, Italy, in 2010, where he is currently working toward the Ph.D. degree with the "E. Piaggio" Research Center.

His research interests are mainly focused on the development of wearable systems and biomedical signal processing and, specifically, on the study of electrodermal activity and eye-tracking systems.

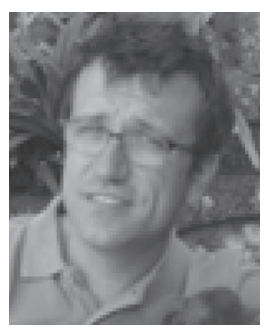

Claudio Gentili received the M.S., M.D., and Ph.D. degrees in psychology from University of Pisa, Pisa, Italy.

He is an Assistant Professor of clinical psychology with University of Pisa. His main fields of research are neurobiology of psychological and personality traits and neurobiology and psychophysiology of anxiety and mood disorders. In particular, he is interested in the neurobiology of social anxiety and social phobia using a functional magnetic resonance facility.

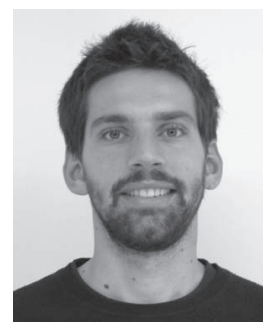

Riccardo Bartolozzi received the B.S. degree in mechanical engineering; the M.S. degree in vehicle engineering, visiting Technical University of $\mathrm{Mu}$ nich, Munich, Germany, for his M.Sc. thesis; and the $\mathrm{Ph} . \mathrm{D}$. degree in vehicle engineering, defending a thesis about dynamic analysis of vehicle systems, from University of Pisa, Pisa, Italy, in 2003, 2006 , and 2011, respectively.

From 2007 to 2011 he was an Assistant Researcher with the Department of Mechanical Engineering, University of Pisa. Since November 2011 he has been an Assistant Researcher with Fraunhofer LBF, Darmstad, Germany. His research interests are vehicle systems dynamics, electric power trains, and real-time simulation for hardware-in-the-loop applications.

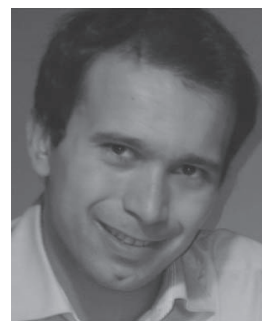

Francesco Bucchi received the Laurea degree (cum laude) in vehicle engineering from University of Pisa, Pisa, Italy, in 2010, where he is currently working toward the Ph.D. degree in mechanical engineering.

Since 2011 he has been a Supervisor of mechanical design and vehicle dynamics with the Formula Student team, University of Pisa. His research interests are in the design and application of magnetorheological fluid devices and in the dynamic simulation of mechanical systems, particularly land vehicles.

Francesco Frendo received the Master's degree (cum laude) in nuclear engineering and the Ph.D. degree in mechanics of materials from University of Pisa, Pisa, Italy, in 1994 and 1998, respectively.

In December 2000 he started his professional career with the Department of Mechanical, Nuclear and Production Engineering, University of Pisa, as an Assistant Professor of machine design. Since December 2011 he has been an Associate Professor of machine design. He has been involved in and has been scientifically responsible for several national and international research projects. He is a coauthor of about 100 scientific papers and an inventor of some international patents.

Dr. Frendo received a National Award from the Italian Association for Stress Analysis in 2000 and two Special Recognition Awards from SAE for papers presented at conferences in 2008 .

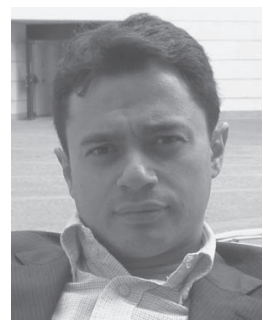

Enzo Pasquale Scilingo (M'10) received the Laurea degree in electronic engineering from University of Pisa, Pisa, Italy, in 1995 and the Ph.D. degree in bioengineering from University of Milan, Milan, Italy, in 1998 .

$\mathrm{He}$ is an Associate Professor of electronic and information bioengineering with University of Pisa. Since 2006 he has been an Assistant Professor with University of Pisa, mainly with the "E. Piaggio" Research Center. He was the coordinator of a European project "PSYCHE-Personalized Monitoring Systems for Care in Mental Health" from 2010 to 2014, and he is a WP leader of other several European projects. He is a coauthor of more than 100 papers, contributions to international conferences, and chapters in international books. His main research interests are in wearable monitoring systems, human-computer interfaces, biomedical and biomechanical signal processing, modeling, control and instrumentation. 